\title{
Is there a risk of malaria transmission in NSW?
}

\section{Ben D. Ewald ${ }^{\mathrm{A}, \mathrm{B}, \mathrm{D}}$, Cameron E. Webb ${ }^{\mathrm{C}}$, David N. Durrheim ${ }^{\mathrm{A}, \mathrm{B}}$ and Richard C. Russell}

${ }^{\mathrm{A}}$ Hunter New England Population Health, Hunter New England Area Health Service

${ }^{\mathrm{B} S c h o o l ~ o f ~ M e d i c a l ~ P r a c t i c e ~ a n d ~ P o p u l a t i o n ~ H e a l t h, ~}$ University of Newcastle

${ }^{\mathrm{C}}$ Department of Medical Entomology, University of Sydney and Institute of Clinical Pathology and Medical Research, Westmead Hospital

DCorresponding author.Email: ben.ewald@newcastle.edu.au

\begin{abstract}
NSW has a putative malaria vector in Anopheles annulipes, and increased numbers of immigrants from malaria endemic countries who may be infective to mosquitoes but asymptomatic. We examine the factors known to influence malaria transmission and conclude that local transmission is possible but unlikely. The public health implications are that there should be systematic screening of immigrants from malaria endemic countries on arrival, and that the public health capacity to identify and respond to a malaria outbreak should be maintained.
\end{abstract}

Australia has been considered malaria free since 1981; however, there are approximately 700 imported cases of malaria reported each year. ${ }^{1,2}$ The most recently documented outbreak of locally acquired malaria occurred in 2002 near Cairns in Queensland with 10 confirmed cases resulting from an infected traveller, who may have become infected in Indonesia or Madagascar. ${ }^{3}$

Australia has been receiving increasing numbers of immigrants from malaria endemic areas, with 9846 people from Africa arriving in 2004-2005 under humanitarian programs. ${ }^{4}$ Of 215 consecutive African refugees screened for malaria at a clinic in Newcastle, $22(10 \%)$ had malarial parasites of at least one species; 21 Plasmodium falciparum, three $P$. malariae, two $P$. ovale, and one $P$. vivax (Davis J, Webber M, personal communication, 2006). Refugee settlement has increased in regional areas where structured screening programs have not previously existed. Unlike returned travellers who have no immunity and thus are symptomatic if infected with malaria parasites, migrants from endemic regions may show no symptoms and go undiagnosed unless specifically tested.

It is therefore important to address the questions: does the presence of the putative vector mosquito An. annulipes in New South Wales (NSW) pose a threat for malaria transmission given the occurrence of imported infections and what are the public health implications?

\section{Background on malaria}

There are four species of human malaria parasites: P. falciparum, $P$. vivax, $P$. ovale and $P$. malariae. The most dangerous is the potentially fatal $P$. falciparum, which together with $P$. vivax accounts for approximately $90 \%$ of cases in Australia. ${ }^{1}$ The incubation period for malaria is generally one to two weeks, but can be longer for certain strains and following chemoprophylaxis. A person with the infection becomes infective to mosquitoes late in the course of the initial illness (from as little as a week to over a month), but can remain periodically infective to mosquitoes for up to a year (P. falciparum) or longer for other malaria species. Not all treatments eradicate the gametocytes, the stage that infects mosquitoes. This means that, with international travel and immigration, there is always a small chance that an infective person will arrive who could infect local Anopheles mosquitoes.

Mosquitoes that feed on gametocyte carriers are not immediately infective, as the malaria parasite undergoes part of its life cycle in the gut wall of the insect and then actively migrates to the salivary glands from where it is injected along with anticoagulant saliva during a bite. The time taken for the mosquito stages to be completed and the mosquito to become infective is referred to as the extrinsic incubation period (sporogony, or the extrinsic cycle) and is highly temperature dependent, ranging from 4 days at $30^{\circ} \mathrm{C}$ to 16 days at $20^{\circ} \mathrm{C}$ (Figure 1). This is of great importance, as at lower temperatures the incubation period may be longer than the average lifespan of the mosquito resulting in few or no infective mosquitoes.

\section{Malaria in NSW}

In Australia, the mosquito known as Anopheles farauti sensu lato is considered the most important vector of malaria. It is principally found in areas north of $19^{\circ}$ latitude in Queensland and north of $15^{\circ}$ latitude in the Northern Territory, although it has been reported in Mackay $\left(21^{\circ} 9^{\prime}\right)$ and Townsville $\left(19^{\circ} 15^{\prime}\right)$, and north of $17^{\circ}$ in the Northern Territory. The area considered at greatest risk of malaria in Australia has been primarily determined by the range of An. farauti s.l., climate supportive of parasite 


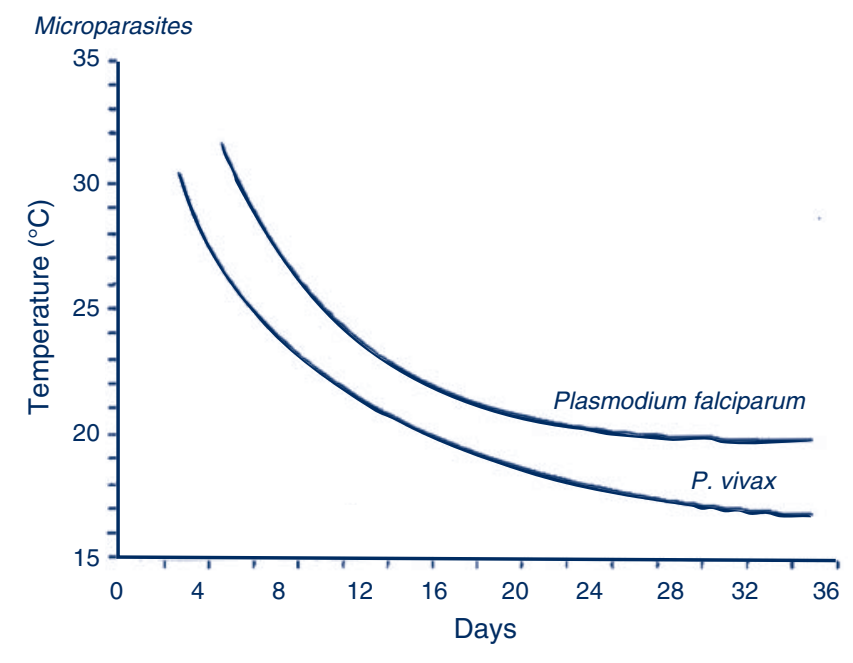

Figure 1. The duration of sporogonic development (latent period) of malaria parasites in the mosquito host (Anopheles spp.) in relation to environmental temperature $\left({ }^{\circ} \mathrm{C}\right) .{ }^{15}$ Used with permission.

transmission and historical records of outbreaks. However, transmission of malaria in Australia has historically occurred as far south as Melbourne in the east and Perth in the west, and a range of Anopheles species occurs throughout the country, although little is known of their respective capacities to transmit Plasmodium parasites under field conditions. ${ }^{5}$

Historically, there has been a small number of locally acquired malaria cases in NSW, thought to be eight in the 20th century, all of them $P$. vivax and most associated with military personnel returning from overseas service with transmission attributed circumstantially to An. annulipes s. $1 .{ }^{5}$ This mosquito is generally the most abundant of the six Anopheles species known to occur in NSW, the others being An. amictus, An. atratipes, An. bancroftii, An. pseudostigmaticus and An. stigmaticus. Laboratory studies have shown An. annulipes s.l. to be capable of transmitting malaria parasites but they have not been found infected in nature. ${ }^{5,6}$ While little is known of the malaria capacity of the remaining species, they are considered relatively rare and unlikely to pose a risk for malaria transmission, although An. amictus can be locally abundant and has been suspected to be involved in malaria transmission in the Northern Territory.

Anopheles annulipes s.l. is a spindly looking grey mosquito of which the wings and legs are mottled with white scales. The larval stages are associated with a range of freshwater habitats and lie flat under the water surface, often in the thin layer of water above algal blooms. This mosquito is typically found in flooded ground pools and, although it is not usually associated with estuarine wetlands, larvae occasionally colonise these brackish water habitats when rainfall has reduced the salinity of the ground pools. The adult mosquitoes may live for up to three weeks and the female mosquitoes take blood meals from humans (as well as other mammals) predominantly from dusk to dawn. Mark-release-recapture experiments on this mosquito species at Griffith, NSW, revealed mean dispersal distances of approximately $1.2 \mathrm{~km}$, although some mosquitoes were found to travel up to $5 \mathrm{~km}{ }^{7}$

In the Australasian region, the mosquitoes called An. annulipes s.l. and An. farauti s.l. are each a group of species, called sibling species, that are morphologically similar but vary in their distribution and biology, and probably in their capacity to transmit Plasmodium species. It is not known which siblings were included in the laboratory studies that demonstrated transmission capacity or were involved in historical field transmission.

\section{Assessing the risk of an outbreak in NSW}

Malaria transmission is dependent on many probabilistic events, such as a mosquito finding someone who is infective (gametocytes present in peripheral blood), the mosquito being capable of being infected and then living long enough to develop sporozoites and thus become infective, and the opportunity of biting a susceptible person and transmitting the parasite. The threats to this process are many: successful treatment of infected people to prevent them becoming infectious to a mosquito; use of personal protection measures against mosquito bites including insect screens, clothing, insecticides and repellents; dry weather that can reduce mosquito longevity; cold weather that prolongs the extrinsic cycle; and the presence of other blood sources and the relatively low density of people in many inland parts of rural and regional NSW where An. annulipes s.l. is more abundant.

Mathematical models offer an understanding of the transmission dynamics of malaria, and while variable values are not available for NSW they can provide a useful framework for considering the risk of local malaria transmission. ${ }^{8}$ The basic reproductive ratio $\left(R_{0}\right)$, the number of new cases of malaria generated by one case introduced into a population of fully susceptible hosts during the duration of the case, may be quantified by multiplying the transmission rate factor from vector to human during the life-span of the vector $\left(T_{H}\right)$ with the transmission rate factor from human to vector during the duration of infection in the human $\left(T_{V}\right)$.

$$
\begin{gathered}
\text { In this model, } T_{H}=V / H a b_{H} L_{V} \\
\text { while } T_{V}=a b_{V} D_{H}
\end{gathered}
$$

where:

- $V$ is the density of vectors

- $H$ is the density of human hosts

- $a$ is the biting rate on the human host per vector, which includes the biting frequency (estimated as the reciprocal of the length of the gonotrophic cycle) and the proportion of blood meals taken on humans 


\section{Box 1. Glossary}

Imported malaria: people arriving with malaria acquired overseas.

Introduced malaria: local transmission from imported cases.

Indigenous malaria: local transmission from other than an imported case.

Established (endemic) malaria: continuing transmission of indigenous malaria.

Receptive areas: places where the climate and suitable vectors could result in established malaria if it was introduced.

Gametocyte: the stage of parasite transferred from humans to mosquitoes.

Sporozoite: the stage of parasite transferred from mosquitoes to humans.

Sensu lato (s.I.): 'in the broad sense' (Latin); when a species name is used to refer to a group of morphologically similar sibling species.

- $b_{H}$ is the proportion of infectious bites on humans that produce a patent infection in humans

- $b_{V}$ is the proportion of bites by susceptible mosquitoes on infected people that produce a patent infection in the vector

- $L_{V}$ is the life expectancy of the vectors

- $D_{H}$ is the duration of infectiousness in the host.

Important assumptions underpinning this model include a lack of immunity in the susceptible human population, which is a robust assumption in NSW and an absence of a parasite-induced effect on vector survival or behaviour., 9,10

$$
\text { Thus } R_{0}=T_{H} \times T_{V}=V / H a^{2} b_{H} b_{V} D_{H} L_{V}
$$

\section{Variables included in a mathematical model for explaining the transmission of malaria}

$V$ : the spatial and temporal abundance of An. annulipes s.l. fluctuates across NSW. The availability of suitable habitat

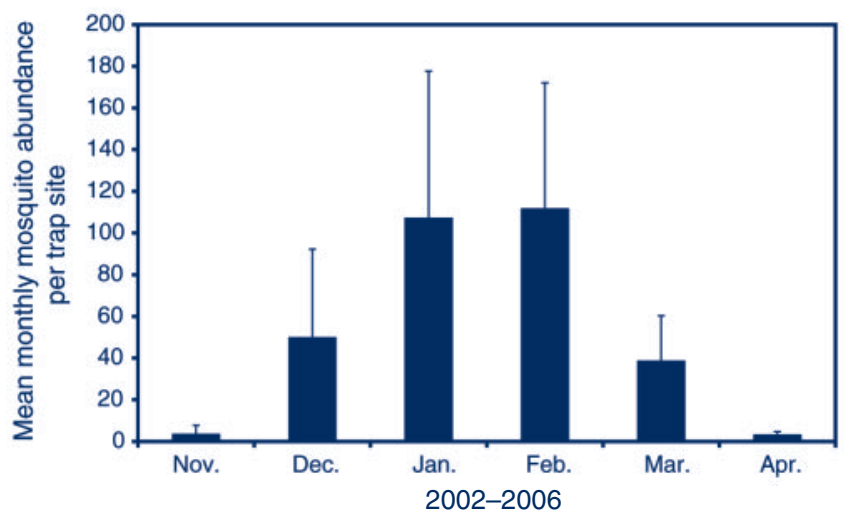

Figure 2. Mean monthly abundance and standard error (SE) of Anopheles annulipes sensu lato at Griffith, NSW; aggregate years 2002-2006 (NSW Health 2006). in Griffith in the inland results in high adult populations in late summer (Figure 2), while in Port Stephens on the coast An. annulipes s.l. is present at relatively low densities (Figure 3) with only a few individual mosquitoes collected in traps that may contain many thousands of mosquitoes dominated by Aedes vigilax and Culex annulirostris.

$H$ : the density of human hosts may be affected by planning decisions if residential developments occur in areas close to productive mosquito habitats or areas of intense mosquito activity. In the inland areas with greatest abundance of An. annulipes, there are generally low to very low human population densities; however, urban sprawl may increase the number of human populations close to mosquito habitats.

$a$ : the number of bites per vector depends on the accessibility of humans to host-seeking female mosquitoes. Biting rates will be influenced by factors such as the use of insect screens, bed nets, insect repellents, presence of alternative blood sources and frequency and nature of nocturnal outdoor activities. The contact between mosquitoes and humans may also be increased when refuge sites for adult mosquitoes, such as heavily vegetated areas, are close to dwellings.

$b_{H}$ : the probability that human infection occurs when bitten by an infected mosquito is generally assumed to be 0.8 to 1.0 .

$b_{V}$ : this variable includes not only the proportion of mosquitoes that become infected while taking a blood meal, but also the delay due to sporogonic development that must occur before they become infective. The duration of sporogony is highly temperature dependent as shown in Figure 1, and is a crucial factor limiting malaria outside the tropics.

$L_{V}$ : to transmit malaria parasites, the female mosquito has a blood meal, lays eggs, and goes looking for another

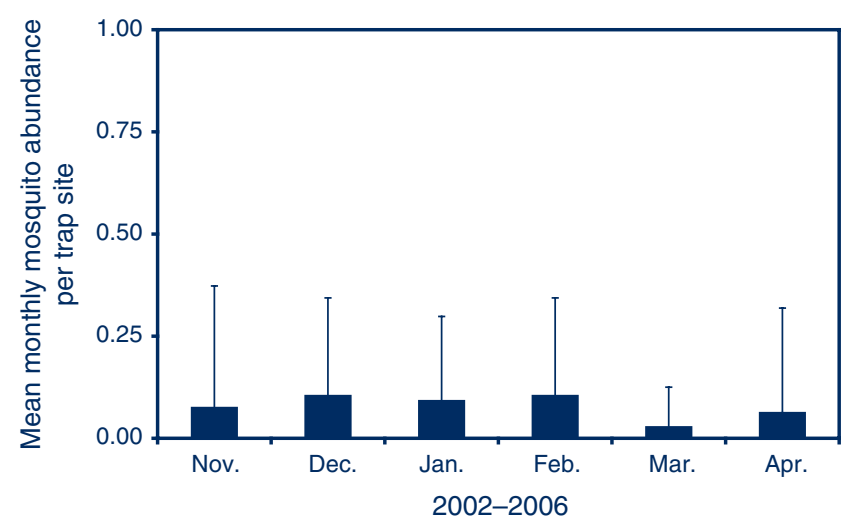

Figure 3. Mean monthly abundance and standard error (SE) of Anopheles annulipes sensu lato at Port Stephens, NSW; aggregate years 2002-2006 (NSW Health 2006). 
meal. The survival of adult mosquitoes is influenced by humidity, temperature and availability of suitable refuge sites (e.g. vegetation). Studies of An. annulipes s.l. at Echuca, Victoria, on the Murray, and Appin, NSW, demonstrated that the oldest mosquitoes were present in summer and late autumn. Based on the ambient temperature at each site, up to $6.6 \%$ of females at Appin and $10.3 \%$ at Echuca lived long enough to potentially become infective. ${ }^{11}$

$D_{H}$ : the duration of infectiveness of a human with malarial gametocytes in their blood depends on the infecting malaria species, whether treatment was provided and what treatment was provided. Untreated $P$. vivax can be infective for $1-2$ years, but $P$. falciparum is generally infective for less than one year. In a malaria-naïve person, the infection will cause symptoms that should prompt early diagnosis by an alert clinician and, hopefully, effective treatment, which interrupts transmission. Such treatment may explain the absence of malaria transmission in NSW in recent decades. People from endemic areas who are tolerant of malaria infection will not have a febrile illness so may not be diagnosed. Unless they are adequately screened, they could remain infective for a prolonged period (Figures 2 and 3).

\section{Scenario}

It is probable that over many years, if Australians keep travelling overseas and immigrants keep arriving, during a period of unusually high mosquito abundance, triggered by major rainfall and during above average temperatures in late summer or early autumn, malaria transmission will occur in NSW. The risk would be further increased in those areas where high levels of human activity occur between dusk and dawn in close proximity to mosquito-breeding habitats and particularly in areas that provide harbourage for adult mosquitoes, including heavily forested parklands within residential or recreational areas.

\section{How long would it take to detect and respond to a malaria outbreak?}

Immigrants tolerant to $P$. falciparum, however, arriving in NSW and carrying gametocytes may be infective to mosquitoes for months or possibly up to or longer than a year. If they were fed on by anopheline mosquitoes capable of transmitting malaria, then the shortest delay before outbreak detection would include the extrinsic cycle of 10-12 days, an incubation period of 7-14 days before infected humans become symptomatic, a diagnostic delay of 7 days before malaria infection is confirmed and a period of 2-4 days for identifying the area requiring appropriate mosquito control and organising appropriate larvicidal and adulticidal treatments: a total of at least 30 days before mosquito control is initiated. During this time, the original infective person would continue to be fed on by mosquitoes. This estimate is similar to the most recent Australian outbreak at Mission Beach, Queensland in 2002, in which the period from infection of the mosquitoes to public health notification was 30-33 days. ${ }^{3}$ If several people were bitten by infective mosquitoes and were all presenting with similar symptoms at the same time, then the diagnostic delay may be reduced. As gametocytes occur relatively late in the course of illness, infection of mosquitoes from secondary cases is unlikely, as supported by experience from North Queensland where despite abundant An. farauti s.l. and higher humidity and temperatures than in NSW, no secondary cases resulted from a local transmission incident. ${ }^{12}$ This outcome may demonstrate the effectiveness of local public health services: conducting careful follow up of cases, particularly regarding exposure to areas where mosquitoes are abundant; excluding the likelihood of local infection during follow-up of confirmed cases; increasing public awareness of measures to reduce exposure to mosquito vectors; and retaining entomological expertise to identify local risks and apply effective larval and adult mosquito control measures.

\section{Climate change}

The possible impact of climate change on malaria in Australia has been discussed elsewhere. ${ }^{13,14}$ The issue is complex, as various mosquito species will be differentially affected by rainfall, temperature and tidal changes. The consensus is that a temperature rise of $1.5^{\circ} \mathrm{C}$ may extend the malaria-receptive zone southward by a couple of hundred kilometres in the Northern Territory and Queensland. This temperature rise would thus extend the area in which malaria vigilance is required but would not pose a public health problem in NSW. The risk of outbreaks after disasters such as cyclones, which are predicted to become more frequent, should be considered in northern Australia, as housing and health-care facilities could be damaged, and potentially require emergency insect control.

\section{Conclusions}

Malaria is not a major health risk in NSW although the possibility of transmission cannot be ruled out completely. Receptivity to malaria will not increase significantly with global warming of $1.5^{\circ} \mathrm{C}$. The high prevalence of malaria found during screening of immigrants in Newcastle suggests that immigrants from malaria-endemic countries should be tested for malaria on arrival in a systematic screening program. In NSW, medical practitioners should maintain a clinical index of suspicion in cases of febrile illness, particularly with multisystem involvement. The public health capacity to respond rapidly and effectively to a malaria outbreak must be ensured.

\section{References}

1. Liu C, Broom AK, Kurucz N, Whelan PI. Communicable Diseases Network Australia National Arbovirus and Malaria Advisory Committee annual report 2004-05. Commun Dis Intell 2005; 29: 341-57. 
2. Longbottom H. Epidemiology of malaria in Australia. Commun Dis Intell 1996; 20: 84-7.

3. Hanna JS, Ritchie SA, Eisen DP, Cooper RD, Brookes DL, Montgomery BL. An outbreak of Plasmodium vivax malaria in far north Queensland, 2002. Med J Aust 2004; 180: 24-8.

4. Department of Immigration and Multicultural Affairs. Immigration Update, July-December 2005. Canberra: Commonwealth of Australia; 2006.

5. Black RH. Malaria in Australia. Service publication 9. School of Public Health and Tropical Medicine, The University of Sydney. Canberra: Australian Government Publishing Service; 1972, p. 222

6. Bryan JH, Russell RC. Australasian malaria vectors. Trans $R$ Soc Trop Med Hyg 1983; 77: 278-9. doi:10.1016/00359203(83)90095-0

7. Bryan JH, Foley DH, Geary M, Carven CTJ. Anopheles annulipes Walker (Diptera: Culicidae) at Griffith, New South Wales. Dispersal of two sibling species. J Aust Entomol Soc 1991; 30: 119-21. doi:10.1111/j.1440-6055.1991.tb00401.x

8. Anderson R, May R. Infectious diseases of humans: dynamics and control. Oxford: Oxford University Press; 1992.
9. Dye C. Vectorial capacity: must we measure all of the components? Parasitol Today 1986; 2: 203-9. doi:10.1016/0169-4758(86)90082-7

10. Dye C. The epidemiological context of vector control. Trans $R$ Soc Trop Med Hyg 1994; 88: 147-9. doi:10.1016/00359203(94)90270-4

11. Russell RC. Seasonal abundance, longevity and population age composition of potential malaria vectors in northern and southern Australia. Aust J Zool 1987; 35: 289-306. doi:10.1071/ZO9870289

12. Brookes DS, Ritchie SA, van den Hurk AF, Fielding JR, Loewenthal MR. Plasmodium vivax malaria acquired in far north Queensland. Med J Aust 1997; 166: 82-3.

13. Bryan JH, Foley DH, Sutherst RW. Malaria transmission and climate change in Australia. Med J Aust 1996; 164: 345-7.

14. Walker J. Malaria in a changing world: an Australian perspective. Int J Parasitol 1998; 28: 947-53. doi:10.1016/S00207519(98)00045-9

15. Macdonald G. The epidemiology and control of malaria. London: Oxford University Press; 1957. 